\title{
Intraperitoneal Delivery of Monoclonal Antibodies: Enhanced Regional Delivery Advantage using Intravenous Unlabeled Anti-Mouse Antibody
}

\author{
RICHARD L. WAHL and SUSAN FISHER
}

\begin{abstract}
Univeristy of Michigan Medical Center, Department of Internal Medicine, Division of Nuclear Medicine,
\end{abstract} Ann Arbor, MI 48109-0028, U.S.A.

(Received 12 February 1987)

\begin{abstract}
Radiolabeled monoclonal antibodies (MAb) delivered intraperitoneally expose cells in contact with peritoneal fluid to considerably higher levels of MAb than if the MAb dose were given intravenously. This regional delivery advantage for intact $\mathrm{MAb}$ is present mainly due to the relatively slow exit of MAb from the peritoneal fluid to the blood. Eventually, following i.p. injection, blood levels of MAb rise resulting in exposure of the animal to high systemic MAb levels and potential toxicity. In this series of experiments, systemic exposure was minimized by the administration of unlabeled goat polyclonal anti-mouse antibody intravenously from $1 \frac{1}{2}$ to $6 \mathrm{~h}$ following i.p. MAb injection. This maneuver results in the formation of immune complexes with their subsequent clearance and dehalogenation by the reticuloendothelial system, thus minimizing systemic MAb exposure. This approach, of increasing systemic clearance of MAb, did not alter intraperitoneal MAb levels and thus significantly increased the regional delivery advantage to the peritoneal cavity by $70-100 \%$. This approach provides an immunologic rationale for the further enhancement of MAb delivery to i.p. foci of malignant disease and may have diagnostic and therapeutic utility.
\end{abstract}

\section{Introduction}

Radiolabeled monoclonal antibodies, by virtue of their specificity for tumor-associated antigens, hold great promise as tumor imaging and therapeutic agents (Mach et al., 1981). These agents have yet to fulfill this promise in part due to their commonly not achieving high tumor/non-tumor ratios following i.v. administation (Strauss et al., 1985). While the use of antibody fragments, particularly the $F\left(a b^{\prime}\right)_{2}$ fragment, has been helpful in this regard, other approaches such as intralymphatic or regional delivery also hold forth promise (Wahl et al., 1983; Weinstein et al., 1982; Epenetos et al., 1982). One such regional approach, the i.p. delivery route, has pharmacologic appeal in that high regional delivery advantages for chemotherapeutic agents and monoclonal antibodies

Correspondence and reprint requests should be addressed to: Richard L. Wahl, M.D., Division of Nuclear Medicine, University of Michigan Medical Center, 1500 East Medical Center Drive, Ann Arbor, MI 48109-0028, U.S.A. [Tel: (313) 936-5384]. can be seen following i.p. administration (Dedrick et al., 1978; Wahl et al., 1985a). These higher levels of antibody in the peritoneal cavity, as compared to the blood, may be useful in treating or diagnosing tumor foci in the peritoneal cavity.

Despite the rational pharmacologic basis for this expectation of enhanced i.p. delivery to i.p. tumors, only limited quantitative preliminary studies of this approach to tumor delivery have been reported. Our preliminary studies in an animal system did not show advantages in terms of antibody delivery to large i.p. tumors (as compared to extraperitoneal tumors in the same animals) following i.p. antibody delivery when assessed several days post-antibody injection (Wahl and Piko, 1985d). Other preliminary studies in man do suggest improved antibody delivery to small i.p. tumor foci following i.p. monoclonal antibody delivery as compared to i.v. monoclonal antibody delivery (Colcher et al., 1986). Recently, we have shown that the i.p. delivery route is superior to the i.v. route in the treatment of i.p. colon cancer using radiolabeled antibodies (Wahl et al., 1987). Thus, i.p. antibody is 
superior to i.v. delivery for i.p. tumor therapy. The lack of enhanced tumor delivery in large tumors, when assessed several days post-injection, may be related to the large size of i.p. tumors, but it is also possible that because the pharmacologic i.p. delivery advantage is somewhat short-lived, mainly in the first $24 \mathrm{~h}$ after the administration of i.p. antibody (Wahl et al., 1985a), that the high initial delivery advantage to i.p. tumors may be masked by later high systemic antibody levels and resultant delivery to non-target tissues by antibody that has entered the bloodstream from the peritoneal cavity.

A potential means to increase the regional delivery advantage following i.p. antibody delivery would be to increase the rate of systemic clearance of antibody without (ideally) accelerating the rate of decline in i.p. antibody levels. One approach capable of accelerating systemic clearance is the use of liposomally entrapped second (anti-mouse) antibody (Begent et al., 1982). A somewhat simpler approach designed to accelerate blood-pool antibody clearance is the use of a polycolonal anti-mouse antibody to accelerate the clearance of mouse monoclonal antibody from the circulation. This approach has been successfully used by Sharkey and co-workers to improve tumor/background ratios following the intravascular administration of radiolabeled polyclonal anti-CEA antibodies to hamsters with CEA-producing colon tumors (Sharkey et al., 1985). This approach with unlabeled polyclonal antibodies (in contrast to efforts with unlabeled monoclonal anti-mouse antibodies) appears to achieve clearance of blood pool radiolabeled anti-tumor antibody through the formation of immune complexes which are then deiodinated by the liver (Sharkey et al., 1985; Wahl et al., 1985b).

In this study, we investigated in normal rats, the ability of polyclonal anti-mouse antibodies administered intravenously to accelerate the blood clearance of an iodinated monoclonal antibody administered intraperitoneally. We also measured the i.v. unlabeled polyclonal anti-mouse effect on peritoneal fluid radioantibody levels following i.p. administration of the radiolabeled antibody. The goal of this work is to determine if the regional delivery advantage of antibodies administered intraperitoneally could be increased in efforts to better diagnose and treat i.p. neoplasms using radiolabeled monoclonals.

\section{Methods}

UPC-10 is an IgG2aK monoclonal antibody purchased in purified form from Litton Bionetics and labeled with ${ }^{125}$ I by the iodogen method (Markwell, 1982). Labeling was performed by mixing $100 \mu \mathrm{g}$ of UPC-10 with $1 \mathrm{mCi}$ of ${ }^{125} \mathrm{I}$ (NEN) in a vial coated with $20 \mu \mathrm{g}$ of iodogen. Yields of $60-80 \%$ proteinbound iodine were typical. The lack of free iodine in the final preparation was confirmed by silica gel thin layer chromatography using a $50 \%$ ethanol $/ 50 \%$ ethyl acetate buffer system (Wahl et al., 1985c).
Polyclonal affinity purified goat anti-mouse antibody was purchased from Cappel (Malvern, Penn.).

Adult, approximately $200 \mathrm{~g}$ female SpragueDawley rats, were sedated with pentobarbital (i.p. $40-70 \mathrm{mg} / \mathrm{kg}$ ) and while under sedation were immobilized in the supine position. A small incision was made in the skin of the abdomen, and under direct visualization, $20 \mathrm{~cm}^{3}$ warmed $\mathrm{pH} 7.2$ phosphate buffered saline, containing $10-50 \mu \mathrm{Ci}$ of ${ }^{125} \mathrm{I}$ UPC-10 $(2-7 \mu \mathrm{g})$, was instilled via a 25 gauge needle into the peritoneal cavity.

Animals were kept sedated for the next approximately $10 \mathrm{~h}$, using repeated small doses of pentobarbital. During this time, sequential blood and peritoneal fluid samples were obtained for radioactivity and weight determination. Blood was removed from the tail vein, while $50-100 \mu \mathrm{L}$ volumes of the peritoneal fluid were removed using a syringe fitted with a 28 gauge needle, under direct visualization.

In one series of experiments, $6 \mathrm{~h}$ following i.p. ${ }^{125} \mathrm{I}$ UPC-10 injection the sedated rats $(n=14)$ were injected with either normal saline intravenously $(250 \mu \mathrm{L})$ or with $250 \mu \mathrm{g}(250 \mu \mathrm{L})$ of the affinity purified goat anti-mouse IgG intravenously. Animals continued to have blood samples obtained (in these animals the peritoneal fluid was not reliably obtained beyond $10 \mathrm{~h}$ post-injection due to absorption) following their regaining consciousness $10 \mathrm{~h}$ post UPC-10 injection, and they were subsequently sacrificed with tissue radioactivity levels and sample weights determined $24 \mathrm{~h}$ following ${ }^{125} \mathrm{I}$ UPC-10 injection using standard tissue handling techniques (Wahl et al., 1984). In another set of experiments, rats received the ${ }^{125}$ I UPC-10 intraperitoneally, but received a $250 \mu \mathrm{g}$ of affinity-purificd goat antimousc antibody dose intravenously $1.5 \mathrm{~h}$ post-intraperitoneal radioantibody injection. Percent $\mathrm{kg}$ dose/g was determined for each time point $(\% \mathrm{~kg}$ dose $/ \mathrm{g}$ is $\%$ injected dose $/ \mathrm{g}$ times the animals weight in $\mathrm{kg}$ ). Radioactive decay was corrected for, and areas under the blood and peritoneal fluid curves were determined in each animal by determining AUC on graphs of individual animal's blood and i.p. fluid radioactivity levels in order to calculate the regional delivery advantage (Rd = AUC IP/AUC blood) (Dedrick et al., 1978; Wahl et al., 1985a). Differences between tissue levels of radioantibody were analyzed using the unpaired Student $t$-test or analysis of variance.

\section{Results}

The blood levels and peritoneal fluid levels of intact ${ }^{125}$ I UPC-10 given by the i.p. route are shown in Fig. 1. Note that in the saline-treated control group blood levels maximize at about $15 \mathrm{~h}$ into the study, and that peritoneal fluid levels change little following injection of the antibody intraperitoneally. Peritoneal fluid is generally totally resorbed (so that sampling is not possible) by $10-15 \mathrm{~h}$ into the study, 


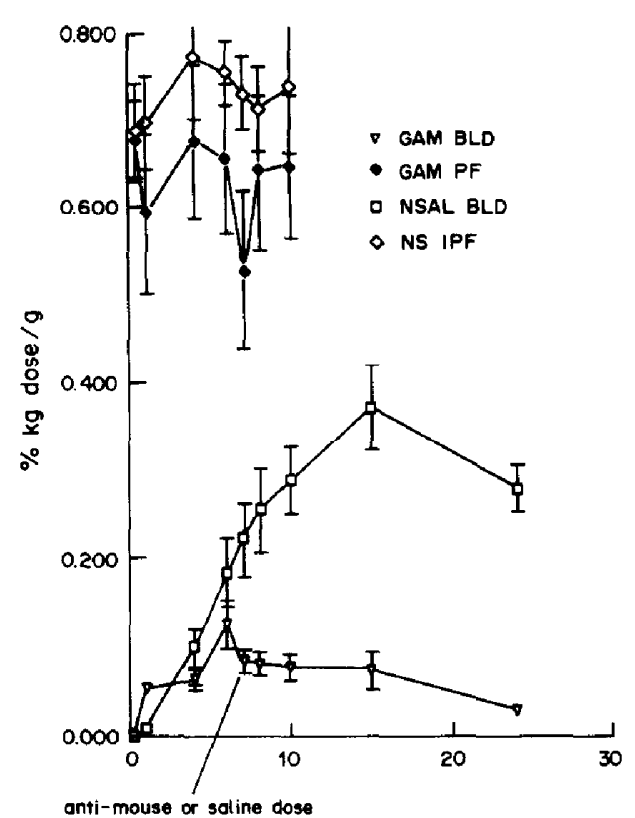

Hours post injection

Fig. 1. Time-activity curve of blood or peritoneal fluid radioactivity levels $(\% \mathrm{~kg}$-dose $/ \mathrm{g}$ ) with or without unlabeled i.v. anti-mouse antibody treatment at $6 \mathrm{~h}$ post-intraperitoneal ${ }^{125}$ I UPC-10 delivery. Note the sharp and persistent decline in UPC-10 blood levels following i.v. polyclonal anti-mouse antibody administration. This is in contrast to the lack of change in peritoneal fluid radioantibody levels. Thus there is a significant enhancement of Rd for the unlabeled goat anti-mouse antibody-treated group $(P<$ 0.025 ). Plots reflect mean of seven animals/group \pm SEM.

so that the 15 and $24 \mathrm{~h}$ time points only show blood activity.

Figure 1 also demonstrates that following the administration of the goat anti-mouse antibody intravenously at $6 \mathrm{~h}$ post-intraperitoneal antibody administration there is a rapid and sustained drop in blood levels of ${ }^{125}$ I UPC-10. Although there is a large difference in blood levels between the saline and anti-mouse groups, this is in contrast to the lack of change in peritoneal fluid levels between the saline and anti-mouse groups. This difference in blood levels is highly significant between the saline and anti-mouse antibody groups $(P<0.01)$ at all times evaluated post the anti-mouse or saline administration as is the enhancement in $\mathrm{Rd}$ at $10.08 \pm 1.29$ vs $6.20 \pm 1.09$ measured from 0 to $10 \mathrm{~h}$ post-injection $(P<0.025)$. The enhanced $\mathrm{Rd}$ was due to this $6-9 \mathrm{~h}$ effect. There was no significant difference between 0 and $6 \mathrm{~h}$ Rd for the saline and the anti-mouse given at $6 \mathrm{~h}$ group. The Rd measured between 6 and $9 \mathrm{~h}$ was $3.79 \pm 0.49$ for the saline group, but increased to $6.97 \pm 0.882$ for the anti-mouse group $(P<0.0005)$.

The tissue distribution of ${ }^{125}$ I UPC-10 activity at sacrifice (Fig. 2) shows that there is a significant drop in activity in nearly all tissues in the animals given polyclonal goat antimouse antibody. The two exceptions were the spleen (where no drop in activity was



Fig. 2. Tissue uptakes of radiolabeled UPC-10 (mean \pm SEM of seven animals/group) $24 \mathrm{~h}$ post ${ }^{125} \mathrm{I}$ UPC- 10 injection intraperitoneally, and $18 \mathrm{~h}$ post i.v. polyclonal goat anti-mouse or normal saline injection. Note the significantly lower $(P<0.005)$ tissue levels in the antibody treated group (except for the spleen where there is no statistically significant difference between saline and anti-mouse treated groups). The thyroids in the saline groups (not shown) had less radioactivity/g than did those of the animals treated with polyclonal goat anti-mouse antibody.

seen), and the unblocked thyroid (not shown on graph) where the cold second antibody groups had significantly $(P<0.01)$ more uptake than the saline treated animals. (Percent $\mathrm{kg}$ dose $/ \mathrm{g}=49.96 \pm 5.46$ SEM for $6 \mathrm{~h}$ cold $\mathrm{Ab}, 74.78 \pm 5.34$ SEM for $1.5 \mathrm{~h}$ cold $\mathrm{Ab}$, and $31.44 \pm 4.02$ for the saline group).

When the polyclonal anti-mouse antibody was given at $1.5 \mathrm{~h}$ following i.p. UPC-10 injection, there was an $\mathrm{Rd}$ of $10.90 \pm 0.668$. This is significantly higher than the Rd seen for i.p. UPC-10 followed by saline at $6 \mathrm{~h}$ (i.v.) $6.2 \pm 1.09(P<0.005)$. There was no significant difference in the $R d 0-9$ achieved by

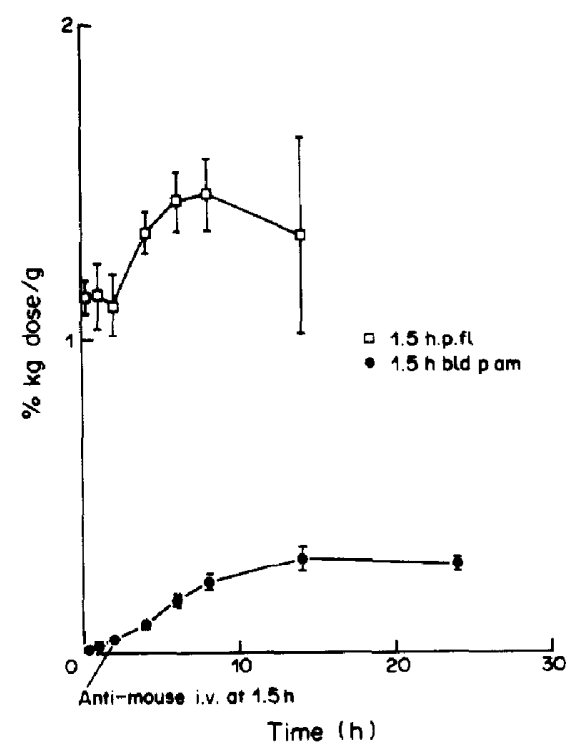

Fig. 3. Time-activity curve of blood and peritoneal fluid radioactivity levels when animals are given $250 \mu \mathrm{g}$ of polyclonal anti-mouse antibody intravenously $1.5 \mathrm{~h}$ following the i.p. administration of $50 \mu \mathrm{Ci}(5-7 \mu \mathrm{g})$ of ${ }^{125} \mathrm{I}$ UPC- 10 . The Rd for $0-9 \mathrm{~h}$ is $10.9 \pm 0.67$. 
giving the polyclonal anti-mouse antibody at 1.5 or $6 \mathrm{~h}$ following i.p. UPC-10 injection (10.90 vs 10.08 ).

\section{Discussion}

Antibody binding kinetic considerations indicate that high local concentrations of antibody bathing tumor cells should produce better tumor uptake than if lower concentrations of antibody are present (Benacerraf and Unanue, 1979). Increasing data supports the i.p. antibody route's superiority over the i.v. in localizing and treating i.p. tumors. Our study shows that the administration of polyclonal anti-mouse antibodies intravenously results in a significantly prolonged drop in blood radioantibody levels, and thus normal tissue levels of radioactivity, without significantly altering i.p. fluid radioantibody levels. This higher antibody concentration in the peritoneal cavity relative to blood levels (regional delivery advantage) should produce better tumor/ non-target uptake for small i.p. tumor foci than would be present if no incremental increase in i.p. concentration was present. Only two normal tissues did not have diminished uptake with polyclonal anti-mouse treatment vs saline treatment: splenic radioantibody levels did not drop with unlabeled anti-mouse treatment relative to spleens in salinetreated animals. Presumedly the spleen takes up immune complexes formed by cold anti-mouse antibody and labeled UPC-10, as does the liver, but the spleen cannot deiodinate them as effectively, resulting in no drop in activity. The thyroid, which was deliberately left unblocked in these studies accumulated significantly more ${ }^{125} \mathrm{I}$ activity/g in those animals treated with cold anti-mouse antibody than did the saline group. This is most compatible with increased availability of free ${ }^{125} I$ to the thyroid due to dehalogenation occurring more rapidly in the cold antibody treated rats.

This unlabeled anti-mouse approach enhances the regional delivery advantage measured to $9 \mathrm{~h}$ of monoclonal antibodies administered intraperitoneally by $70-100 \%$ fold over saline treated control animals. We cannot yet state with certainty whether this approach will enhance the relative targeting of tumor-specitic monoclonals to foci of human cancer situated intraperitoneally. While this is most probable, animal model system of human i.p. carcinomatosis are generally located in the mouse, and do not easily allow for the testing of the hypothesis in the tumor-bearing animals due to difficulty with the anti-mouse antibodies cross reacting with circulating mouse IGs.

It is possible that higher doses of unlabeled antimouse antibody might further enhance Rd. However, there might be difficulties with that approach (if excessive polyclonal anti-mouse antibody is given) as tumor uptake of radioantibody potentially might be diminished. Thus, determination of the optimal dose of unlabeled antibody would be essential in each system studied.

In conclusion, our study demonstrates that while a major regional delivery advantage exists for an intact murine monoclonal IgG antibody given intraperitoneally, this delivery advantage can be significantly enhanced by the systemic administration of polyclonal anti-mouse antibodies, allowing for the rapid clearance of ${ }^{125}$ I UPC-10 from the blood after it diffuses there from the peritoneal cavity. This approach of accelerated systemic clearance provides a pharmacokinetic rationale for improving regional delivery to any body cavity and may be particularly useful in the diagnosis and treatment of i.p. carcinomas such as ovarian and colon cancers.

Acknowledgements - This investigation was supported by PHS Grant No. CA41531-01 and CA33802-04 awarded by the National Cancer Institute, DHHS.

Thanks to Mrs Michele Bell for excellent secretarial assistance.

\section{References}

Benacerraf B. and Unanue E. (1979) Immunology: principle and phenomenology. In Textbook of Immunology, pp. 1-11. Williams and Wilkins, Baltimore, Md.

Begent R. H., Keep P. A. and Green A. J. et al. (1982) Liposomally entrapped second antibody improves tumor imaging with radiolabeled (first) anti-tumor antibody. Lancet 1(8332), 1047-1048.

Colcher D., Esteban J. and Carrasquillo J. E. et al. (1986) Comparison of route of administration of radiolabeled monoclonal antibodies in patients with colorectal cancer. J. Nucl. Med. 27, 902.

Dedrick R. L., Myers C. E. and Bungay P. M. et al. (1978) Pharmacokinetic rationale for peritoneal drug administration in the treatment of ovarian cancer. Cancer Treat. Rep. 62, 1-11.

Epenetos A. A., Britton E. K. and Mather S. et al. (1982) Targeting of I-123 labeled tumor-associated monoclonal antibodies to ovarian, breast, and gastrointestinal tumors. Lancet 2(8306), 999-1005.

Mach J. P. Buchegge F. and Forni M. et al. (1981) Use of radiolabeled monoclonal anti-CEA antibodies for the detection of human carcinoma by external photoscanning and tomoscintigraphy. Immunol. Today 2, 239-249.

Markwell M. A. K. (1982) A new solid-state reagent to iodinate proteins. I. Conditions for the efficient labeling of antiserum. Anal. Biochem. 125, 427-432.

Sharkey R. M., Primus F. J. and Goldenberg D. M. (1985) Second antibody clearance of radiolabeled antibody in cancer radioimmunodetection. PNAS-USA 81, 2843-2846.

Strauss H. W., Carrasquillo J. A. and Larson S. M. (1985) Antibody imaging: The smoke, the fire and the false alarm. Int. I. Nucl. Med. Biol. 12, 401-403.

Wahl R. L., Parker C. W. and Philpott G. W. (1983) Improved radioimaging and tumor localization with monoclonal $\mathrm{F}\left(\mathrm{ab}^{\prime}\right)_{2}$. J. Nucl. Med. 24, 316-325.

Wahl R. L., Sherman P. and Fisher S. (1984) The effect of specimen processing on radiolabeled monoclonal antibody biodistribution. Eur. J. Nucl. Med. 9, 382-384.

Wahl R. L., Geatti O. and Fisher S. (1985a) Intraperitoneal delivery of monoclonal antibodies: Influence of class and fragmentation of kinetics and intraperitoneal dosing advantage. J. Nucl. Med. 26, P114. 
Wahl R. L., Laino L., Jackson G., Fisher S. and Beierwaltes W. H. (1985b) Influence of unlabeled monoclonal antimouse antibody on the clearance rate of radiolabeled mouse monoclonal antibody. J. Nucl. Med. 26, P66-67.

Wahl R. L., Liebert M., Carey J. E. and Jackson G. (1985c) Quality control of radiolabeled monoclonal antibodies: Immunologic and radiochemical. Cancer Drug Delivery 2 , 236.

Wahl R. L. and Piko C. (1985d) Intraperitoneal (IP) delivery of radiolabeled monoclonal antibody to IP. induced xenografts of human ovarian cancers. Proc. American Association for Cancer Res. 26, 298.

Wahl R. L., Liebert M., Fisher S. and Boland R. (1987) Enhanced radioimmunotherapy of intraperitoneal human colon cancer xenografts by intraperitoneal monoclonal antibody delivery. Proc. American Association for Cancer Res. 28, 438.

Weinstein J. N., Parker R. J., Keenan A. M., Dower S. K., Morse H. C. and Sieber S. M. (1982) Monoclonal antibodies in the lymphatics: Toward the diagnosis and therapy of tumor metastases. Science 218, 1334-1337. 